\title{
A Giant Retroperitoneal Liposarcoma Encasing the Entire Left Kidney and Adherent to Adjacent Structures: A Case Report
}

\author{
Sung Don $\mathrm{Oh}^{\mathrm{a}}$ Sung Jin $\mathrm{Oh}^{\mathrm{a}}$ Byoung Jo Suh ${ }^{\mathrm{a}}$ Jin Yong Shin ${ }^{\mathrm{a}}$ \\ $\mathrm{Cheol} \mathrm{Kyu} \mathrm{Oh}^{\mathrm{b}}$ Jong Kwon Park ${ }^{\mathrm{a}}$ Yeon Mee Kim ${ }^{\mathrm{c}}$ Bo Mi Kim ${ }^{c}$ \\ ${ }^{a}$ Department of Surgery, Haeundae Paik Hospital, Inje University College of Medicine, \\ Busan, South Korea; ${ }^{b}$ Department of Urology, Haeundae Paik Hospital, Inje University \\ College of Medicine, Busan, South Korea; ${ }^{C}$ Department of Pathology, Haeundae Paik \\ Hospital, Inje University College of Medicine, Busan, South Korea
}

\section{Keywords}

Giant retroperitoneal liposarcoma $\cdot$ Organ preservation · Prognosis

\begin{abstract}
Retroperitoneal liposarcoma is a rare tumor. The dimension and weight of liposarcoma are variable; those over $20 \mathrm{~kg}$ are called 'giant liposarcoma'. Herein, we report giant retroperitoneal liposarcoma measuring $45 \mathrm{~cm}$ in diameter and $25 \mathrm{~kg}$ in weight encasing the entire left kidney and adherent to adjacent structures. A 71-year-old woman presented for a regular checkup. Image study revealed a huge mass probably indicative of retroperitoneal liposarcoma encasing the entire left kidney and adherent to adjacent structures. We performed an organ-preserving surgical removal. The pathologic report was liposarcoma. At postoperative month 16, a follow-up CT revealed a locally recurrent tumor. The patient underwent surgical removal of the newly discovered mass. After the second surgery, the patient underwent regular follow-up CT for approximately 12 months, and to date, there has been no evidence of tumor recurrence. High-grade liposarcoma shows sensitivity to radiation therapy. However, the toxic effect of radiation therapy limits this option by treatment modality. The use of chemotherapy is also controversial. As a result, complete resection is the gold standard
\end{abstract}


Oh et al:: A Giant Retroperitoneal Liposarcoma Encasing the Entire Left Kidney and Adherent to Adjacent Structures: A Case Report

treatment. Here, we report a giant retroperitoneal liposarcoma encasing the entire left kidney and adherent to adjacent structures, describe successful organ-preserving surgical removal and discuss prognosis.

\section{Introduction}

Soft tissue sarcomas are very rare tumors; however, there are many histologic subtypes. Among those subtypes, liposarcoma is one of the most common soft tissue sarcomas [1]. Furthermore, among sarcomas originating within the retroperitoneum, which constitute 10$15 \%$ of all soft tissue sarcomas, liposarcomas are the most common histologic type, accounting for $41 \%$ of these tumors $[2,3]$. Liposarcomas are generally located in the head, neck, trunk, mediastinum, upper and lower extremities, gastrointestinal tract, and retroperitoneum [4]. They commonly occur in patients aged 40-60 years, and men and women are equally affected [4].

The dimensions and weight of liposarcomas are variable; those over $20 \mathrm{~kg}$ are called 'giant liposarcomas' and are extremely rare [4].

Herein, we report a giant retroperitoneal liposarcoma weighing $25.0 \mathrm{~kg}$, encasing the entire left kidney and adherent to adjacent structures which was successfully removed with kidney and aorta preserving.

\section{Case Report}

A 71-year-old woman presented with complaint of progressive abdominal distension. The ultrasonography revealed a huge retroperitoneal or mesenchymal mass occupying the entire abdomen. The patient had not experienced any symptoms, such as abdominal pain, nausea, vomiting, constipation, dyspepsia, or dyspnea. She had a history of hypertension only.

The patient was admitted to the hospital and underwent contrast-enhanced computed tomography (CT) of the abdomen. The scan revealed a huge fatty mass originating from the retroperitoneum probably indicative of retroperitoneal liposarcoma. The CT scan also showed tumor encasement of the entire left kidney. Septations and solid portions were observed within the mass. The spleen was pushed anteriorly and the small bowel was deviated to the right side of the intra-abdominal space by the mass (fig. 1).

Because the patient was old, we decided to attempt organ-preserving surgery for the removal of the tumor to minimize the morbidity. The mass was approached by making a midline incision. Adherence of the mass to the diaphragm, stomach, spleen, pancreas, and aorta could be observed. The greatest difficulty was that the tumor was encasing the entire left kidney and adherent to the aorta. Although the entire left kidney was encased with the huge tumor, neither the renal parenchyma nor the ureter was invaded. We successfully performed a salvage of the left kidney by wide excision and separated the tumor from the aorta by shaving it away, thus preserving both kidney and the aorta. The specimen measured 45.0 $\times 30.0 \times 11.0 \mathrm{~cm}$ and weighted $25.0 \mathrm{~kg}$ (fig. 2). Microscopic examination showed a combined type of liposarcoma (tumor component: well-differentiated liposarcoma, more than 95\%, myxoid liposarcoma, less than 5\%). An average of 1-2 mitotic figures were noted per highpower field. According to the grading system of the French Federation of Cancer Centers Sarcoma Group, the tumor was classified as grade 1 (fig. 3). 
Oh et al.: A Giant Retroperitoneal Liposarcoma Encasing the Entire Left Kidney and Adherent to Adjacent Structures: A Case Report

The patient was discharged after an uneventful postoperative hospital stay. She underwent regular follow-up examinations for 16 months after the operation. At 16 months, a follow-up CT scan revealed a newly defined low-density soft tissue mass in the aortocaval and preaortocaval area, measuring $3.5 \times 1.8 \times 4.3 \mathrm{~cm}$, suggesting a locally recurrent tumor in the retroperitoneum. We performed positron emission tomography (PET), which revealed tumor recurrence in the retroperitoneum. The patient underwent surgical removal of the newly discovered mass. The histologic diagnosis was well-differentiated liposarcoma of sclerosing type. Fluorescence in situ hybridization showed MDM2 amplification, which was consistent with a diagnosis of well-differentiated liposarcoma. After the second surgery, the patient underwent regular follow-up CT scans for approximately 12 months, and to date, there has been no evidence of tumor recurrence

\section{Discussion}

Liposarcoma is one of the most common soft tissue sarcomas, constituting approximately $20 \%$ of cancers within that group [5]. Between 10 and $15 \%$ of soft tissue sarcomas originate within the retroperitoneal space, and the most common type among these is liposarcoma [3]. However, liposarcoma is very rare overall, accounting for $0.2-0.3 \%$ of all malignancies [2].

Multiple factors, such as site and depth of origin, margin involvement after resection, and histologic grade affect survival rates for patients with liposarcoma. Distant metastasis relates to the tumor size. If the size of the tumor is less than $2.5 \mathrm{~cm}$, the rate of metastasis at 5 years is approximately $3 \%$. On the other hand, the rate of metastasis at 5 years is between 55 and $60 \%$ in cases of tumors larger than $20 \mathrm{~cm} \mathrm{[6].} \mathrm{Retroperitoneal} \mathrm{origin} \mathrm{is} \mathrm{a} \mathrm{poor} \mathrm{prog-}$ nostic factor. This is because the retroperitoneal space allows the tumor to grow to a large size before the appearance of clinical signs and symptoms [6]. Therefore, the tumor is often diagnosed at the advanced stage. Resection margin involvement also affects prognosis. Cases with involved resection margins are characterized by a tendency towards local recurrence [7]. The histologic subtype was significantly associated with recurrence $[8,9]$. The five recognized histologic types are the well-differentiated, myxoid, round cell, pleomorphic, and dedifferentiated type. The well-differentiated type has good prognosis, with 5-year survival rates of approximately $90 \%$ [9]. The myxoid type has a less satisfactory progression, and often displays early recurrence [5]. The 5-year survival rates of the remainder of the histologic types are poor.

High-grade liposarcoma shows sensitivity to radiation therapy. However, the toxic effects of radiation therapy limit this option by primary treatment modality [4]. The use of chemotherapy is also controversial. Research has documented little benefit from adjuvant chemotherapy in well-differentiated low-grade tumors, and partial responses in high-grade diseases in up to $50 \%$ of patients, with increased overall survival [9]. As a result, complete surgical resection is the gold standard treatment, which might be curative. In many cases, combined resection of involved organs and vasculatures is required to achieve complete resection. Therefore, the most commonly sacrificed organ is the kidney, followed by the colon, pancreas, major vasculature, and spleen [10].

In this study, we reported on a giant retroperitoneal liposarcoma encasing the entire left kidney and adherent to adjacent structures. Furthermore, we described successful organpreserving surgical removal and discussed the prognosis. Although there has been no evi- 
Oh et al:: A Giant Retroperitoneal Liposarcoma Encasing the Entire Left Kidney and Adherent to Adjacent Structures: A Case Report

dence of recurrence to date, we will continue to observe our patient closely for recurrence, as in other previously published reports.

\section{Statement of Ethics}

Written informed consent for publication of this paper was obtained from the patient.

\section{Disclosure Statement}

The authors have nothing to declare.

\section{References}

1 Mack TM: Sarcomas and other malignancies of soft tissue, retroperitoneum, peritoneum, pleura, heart, mediastinum, and spleen. Cancer 1995;75:211-244.

2 Shahaji C, Amit P, Prashant P, Sachin T: Giant retroperitoneal liposarcoma: a case report. Case Rep Oncol Med 2012;4:1-2.

3 Caizzone A, Saladino E, Fleres F, et al: Giant retroperitoneal liposarcoma: case report and review of the literature. Int J Surg Case Rep 2015;9:23-26.

4 Leao P, Vilaca S, Oliveira M, Falcao J: Giant recurrent retroperitoneal liposarcoma initially presenting as inguinal hernia: review of literature. Int J Surg Case Rep 2012;3:103-106.

-5 Ki EY, Park ST, Park JS, Hur SY: A huge retroperitoneal liposarcoma: case report. Eur J Gynaecol Oncol 2012;33:318-320.

-6 McCallum OJ, Bruke JJ II, Childs AJ, Ferro A, Gallup DG: Retroperitoneal liposarcoma weighing over one hundred pounds with review of the literature. Gynecol Oncol 2006;103:1152-1154.

7 Kirane A, Crago AM: The importance of surgical margins in retroperitoneal sarcoma. J Surg Oncol 2016;113:270-276.

-8 Nijhuis PH, Sars PR, Plaat BE, Molenaar WM, Sluiter WJ, Hoekstra HJ: Clinico-pathological data and prognostic factors in completely resected AJCC stage I-III liposarcomas. Ann Surg Oncol 2000;7:535543.

99 Singer S, Antonescu CR, Riedel E, Brennan MF: Histologic subtype and margin of resection predict pattern of recurrence and survival for retroperitoneal liposarcoma. Ann Surg 2003;238:52-65.

10 Jaques DP, Coit DG, Hajdu SI, Brennan MF: Management of primary and recurrent soft-tissue sarcoma of the retroperitoneum. Ann Surg 1990;212:51-59. 


\section{Case Reports in Oncology}

\begin{tabular}{l|l}
\hline Case Rep Oncol 2016;9:368-372 \\
\hline $10.1159 / 000447488$ & $\begin{array}{l}\text { C 2016 The Author(s). Published by S. Karger AG, Basel } \\
\text { www.karger.com/cro }\end{array}$ \\
\hline
\end{tabular}

Oh et al:: A Giant Retroperitoneal Liposarcoma Encasing the Entire Left Kidney and Adherent to Adjacent Structures: A Case Report
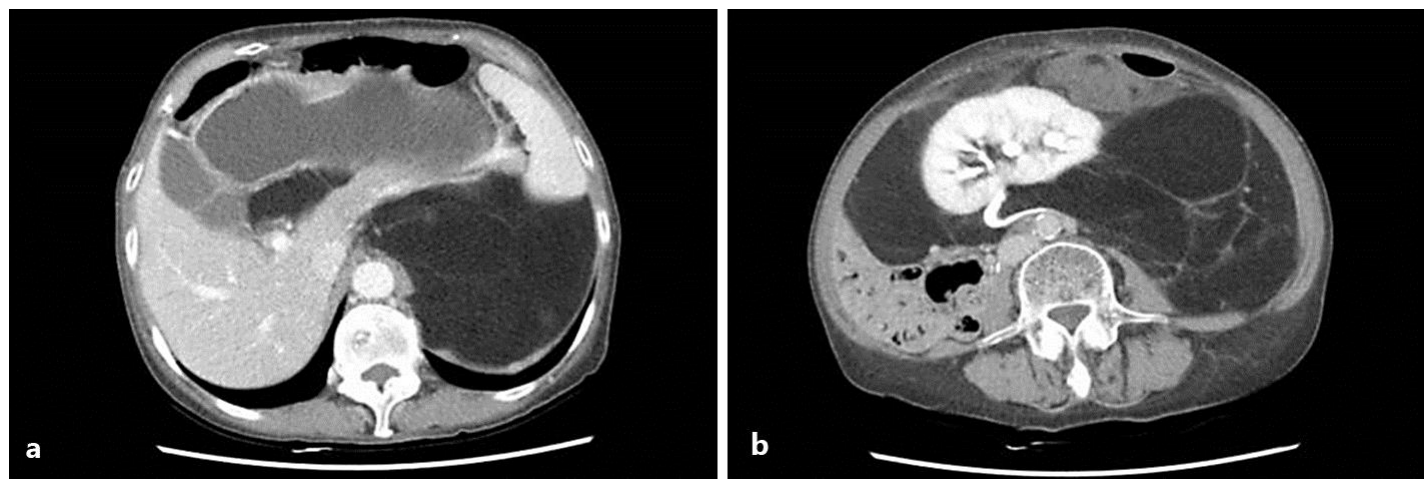

Fig. 1. Septations and solid portions are observed within the mass. a The spleen is pushed anteriorly and the small bowel is deviated to the right side of the intra-abdominal space by the mass. $\mathbf{b}$ CT scan showing a huge fatty mass originating from the retroperitoneum and encasing the entire left kidney.
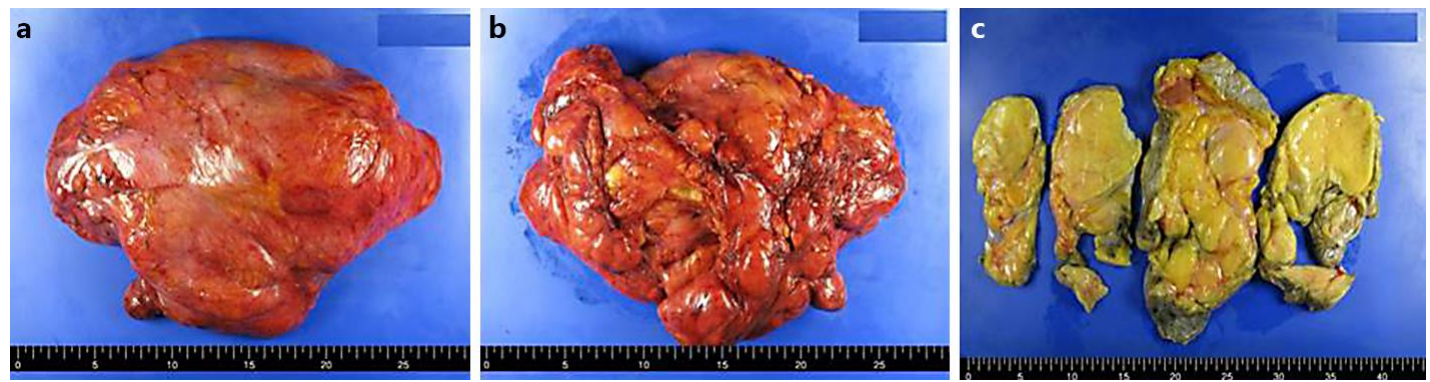

Fig. 2. a-c Images of the surgically removed specimens and multiple cut sections. The specimen measured $45.0 \times 30.0 \times 11.0 \mathrm{~cm}$ and weighed $25.0 \mathrm{~kg}$.
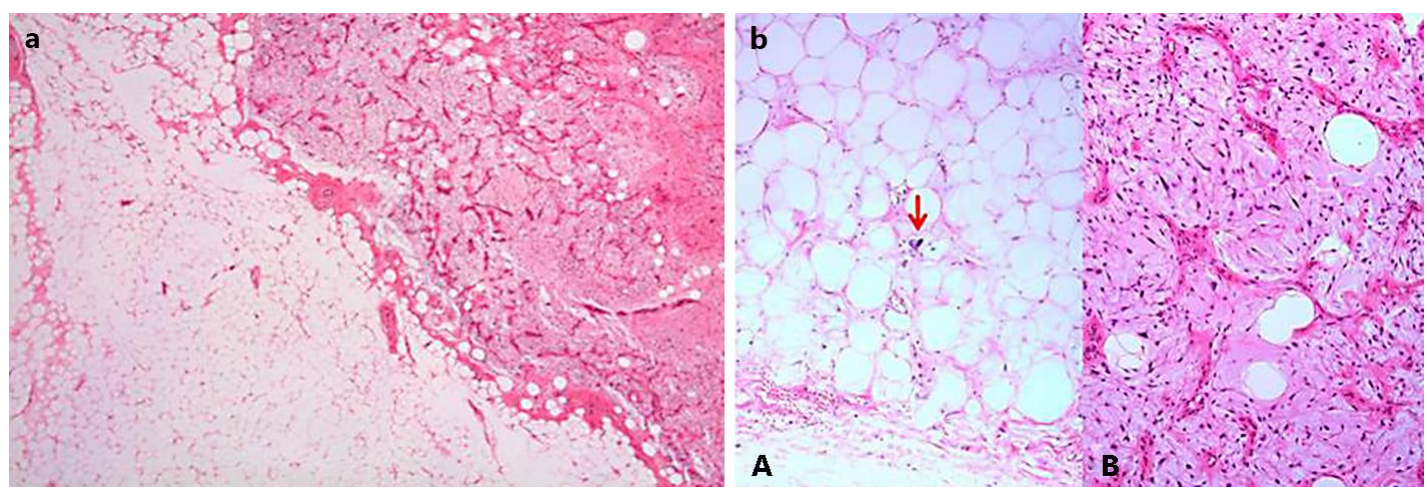

Fig. 3. Low-power view demonstrates well-differentiated liposarcoma and myxoid liposarcoma. a A lipoma-like lesion, classified as atypical lipomatous tumor/well-differentiated liposarcoma, is shown. b In the myxoid liposarcoma area, the tumor displays a nodular growth pattern with prominent myxoid stroma and arborizing vasculature. Hematoxylin and eosin staining. Magnification $\times 40$. In the high-power view of the well-differentiated liposarcoma, the size variation of adipocytes is noted. The red arrow indicates atypical stromal cells with nuclear hyperchromatism. A In the myxoid liposarcoma area, there is a mixture of lipocytes and nonlipogenic mesenchymal cells with a 'chicken-wire' capillary vasculature and myxoid stroma. B Hematoxylin and eosin staining. Magnification $\times 20$. 